\title{
Philosophiques
}

Mark Steiner, The Applicability of Mathematics as a Philosophical Problem, Cambridge, Mass., Cambridge University Press, 1998, 215 pages.

\section{Yvon Gauthier}

Volume 30, numéro 1, printemps 2003

Bernard Bolzano. Philosophie de la logique et théorie de la connaissance

URI : https://id.erudit.org/iderudit/007745ar

DOI : https://doi.org/10.7202/007745ar

Aller au sommaire du numéro

Éditeur(s)

Société de philosophie du Québec

ISSN

0316-2923 (imprimé)

1492-1391 (numérique)

Découvrir la revue

Citer ce compte rendu

Gauthier, Y. (2003). Compte rendu de [Mark Steiner, The Applicability of Mathematics as a Philosophical Problem, Cambridge, Mass., Cambridge University Press, 1998, 215 pages.] Philosophiques, 30(1), 266-267.

https://doi.org/10.7202/007745ar d'utilisation que vous pouvez consulter en ligne.

https://apropos.erudit.org/fr/usagers/politique-dutilisation/ 
Le mérite essentiel de l'ouvrage de l'auteure se situe du côté de la fine analyse historique de certaines théories du champ, de Maxwell à Einstein et Weinberg. Elle n'a pas réussi cependant à fonder la thèse principale de son ouvrage, la surdétermination des théories physiques par les structures mathématiques (l'appareil analytique), sur l'analyse aussi fine d'une perspective unifiée dans les fondements logico-mathématiques (formels) de la pratique physicienne.

YVON GAUTHIER

Université de Montréal

\section{Mark Steiner, The Applicability of Mathematics as a Philosophical Problem, Cambridge, Mass., Cambridge University Press, 1998, 215 pages.}

Ce petit ouvrage propose une solution au problème de l'applicabilité des mathématiques. L'auteur privilégie la physique, en particulier la mécanique quantique, comme lieu d'application des concepts mathématiques et il baptise sa solution d'anthropocentrique (ou formaliste) qu'il veut opposer à la thèse naturaliste d'inspiration quinéenne. L'anthropocentrisme de Steiner suppose que l'homme occupe une place centrale dans l'univers physique, place que le naturalisme concevrait comme insignifiante (p. 25). L'épistémologie anthropocentriste insiste sur le caractère formel des concepts mathématiques qui seraient directement applicables au réel physique, parce que ce sont des créations humaines.

Cette version faible du constructivisme emprunte des moyens, allant du platonisme au formalisme, qui ne sont pas toujours convergents. Par exemple, dans un premier chapitre l'auteur fait le détour "platoniste" par l'arithmétique du second ordre de Frege avec le principe huméen de l'égalité extensionnelle — un nombre appartient à la fois à $F$ et à $G$, ssi $F$ et $G$ sont des concepts équinumériques — pour garantir l'applicabilité sémantique des mathématiques. Mais dans le cas frégéen, la garantie passe par l'existence des ensembles au sens de la sémantique ensembliste, en particulier l'existence de l'ensemble infini des nombres naturels de cardinalité ${ }_{-0}$, ce qui ne semble guère anthropocentrique de prime abord, l'appartenance des objets physiques à des ensembles s'apparentant à la participation platonicienne des objets sensibles aux Formes ou Idées du ciel intelligible. Que les objets physiques soient subsumés dans des ensembles n'est pas en soi une critique valable du naturalisme et l'auteur a beau se défendre de platonisme, sa stratégie frégéenne ne fait qu'accentuer le réalisme latent d'un structuralisme larvé. Je dis détour, parce que la voie directe est évidemment la théorie des modèles, si l'on veut une sémantique pour quelque système formel que ce soit. Frege n'avait pas la notion de modèle, mais Hilbert l'avait dès 1899 avec le modèle arithmétique des axiomes de la géométrie et c'est cette notion qu'il exploite pour sa théorie des fondements de la physique dans l'article séminal «Ueber die Grundlagen der Quantenmechanik », Mathematische Annalen (1928, 1-30) écrit en collaboration avec J. von Neumann et L. Nordheim. Ici les notions d'appareil analytique "analytischer Apparat " et de conditions de réalité "Realitätsbedingungen " tiennent lieu de formalisme logico-mathématique et de modèles. Von Neumann s'est servi abondamment de ses concepts dans son classique de 1932 sur les fondements mathématiques de la mécanique quantique : vu que l'appareil analytique ne saurait être 
catégorique ou canonique ( avec interprétation unique), il y une multiplicité de modèles, standard et non standard. L'épistémologie constructiviste fait ainsi l'économie de ces détours que l'auteur multiplie avec ses analogies pythagoriciennes (chapitre 3 et 4 ) et son structuralisme formel. Le principe de maximalité pour l'espace de Hilbert que l'auteur invoque ( p. 38 et ss. ) devient dans ce contexte un requisit philosophique qui ne peut être justifié formellement, mais dont on pourrait trouver l'équivalent formel en postulant comme l'a fait von Neumann que les observables (quantités physiques) sont en bijection avec les opérateurs hermitiques de l'espace de Hilbert.

Malgré son style indirect, l'analyse de l'auteur est fort pertinente dans les chapitres consacrés à la mécanique quantique (chapitres 5 et 6). L'auteur offre, par exemple, une dérivation non physique de la mécanique quantique (appendice A ). À part l'élégance de cette "quantisation formelle", l'auteur semble oublier que l'espace de Hilbert, baptisé par von Neumann, a une genèse mathématique bien définie. Ce sont en effet les travaux de Hilbert en théorie des équations intégrales qui ont permis d'en arriver à une théorie des probabilités sur l'espace fonctionnel $\mathrm{L}^{2}$ (classe d'équivalence des fonctions de carré intégrable) où la notion de probabilité comme carré de valeur absolue de la fonction d'onde $\left.I_{-}\right|^{2}$ trouve sa justification formelle. La dérivation non physique du formalisme rend possible son interprétation sans que celui-ci ne devienne canonique et des espaces plus généraux ( e.g. espace de Fock ) peuvent servir de cadre formel à la mécanique quantique - on trouvera des précisions sur le formalisme de l'espace de Hilbert dans Logique interne. Modèles et applications, Paris/Montréal, Diderot/Modulo, 1997, pp. 138-141. Le mystère dont parle l'auteur au chapitre 6 de son livre «Formalist reasoning : the mystery of quantization » se dissipe si l'on tient compte du fait que des formulations distinctes de la mécanique quantique, de Schrödinger à Heisenberg et à Dirac, sont équivalentes ou que les théories de jauge, de Weyl à Yang - Mills, sont tributaires d'une construction a priori, comme le dirait Weyl lui-même. L'auteur aurait pu remarquer ici dans son traitement succinct que l'invariance de jauge locale introduite par Weyl avait un motif géométrique, le théorème de Pythagore dans l'infiniment petit pour la géométrie riemannienne. L'auteur n'aborde pas la Relativité Générale, mais il aurait pu y retrouver la même motivation dans la définition de l'élément métrique d'une variété riemannienne.

Steiner s'est attaché à décrire la théorie physique du point de vue de son contenu physique en montrant comment ce contenu était surdéterminé par le formalisme mathématique, un peu dans le même sens que Margaret Morrison dans son ouvrage Unifiyng Scientific Theories. Physical Concepts and Mathematical Structures (Cambridge, Cambridge University Press, 2000). Mais pas plus que Morrison, Steiner n'a produit une analyse comparable des structures mathématiques qu'il met en jeu. Je pense que la raison principale de ce demi-succès tient à la perspective plutôt hétéroclite qu'il a adoptée dans son ouvrage. L'anthropocentrisme «formel » qu'il oppose à un naturalisme «informe » se nourrit d'une tradition philosophique hétérogène. Eûtil opté pour une attitude fondationnelle résolument constructiviste à la Hilbert, sa thèse sur l'applicabilité des mathématiques en eût été plus unifiée et son analyse plus convaincante, analyse qui reste fort utile quand ce ne serait que parce qu'elle pose le problème de la logique interne des théories physiques avec une belle acuité.

YVON GAUTHIER

Université de Montréal 